\title{
Spatial spectrum of temperature fluctuations in buoyancy driven chaotic and turbulent atmosphere
}

\author{
A. Bershadskii \\ ICAR, P.O. Box 31155, Jerusalem 91000, Israel \\ bershads@gmail.com
}

\begin{abstract}
It is shown, using results of direct numerical simulations, laboratory experiments, measurements in the atmospheric boundary layer, in troposphere, in stratosphere, and the satellite infrared radiances data that in many cases the temperature fluctuations in buoyancy driven chaotic and turbulent atmosphere can be well described by the distributed chaos approach based on the Bolgiano-Obukhov phenomenology. A possibility of analogous consideration of the atmospheric dynamics on other planets (Venus, Mars, Jupiter and Saturn) has been briefly discussed using the data obtained with the Phoenix lander and the radio and infrared observations.
\end{abstract}

\section{INRODUCTION}

At free convection the fluid or gas are driven by the density fluctuations produced by temperature (humidity) differences (see, for instance, Ref. [1]). The mean flows in forced convection (especially with a wind shear) can drastically change the fluid dynamics and result in non-universality of the observed spectra of the atmospheric temperature fluctuations. On the other hand, one can expect that the free convection can be characterized by universal types of the spectra. Therefore, the atmospheric free convection can be used for the fundamental studies of the buoyancy driven turbulence. In many cases the universal types of the spectra can be also observed in the buoyancy driven convection despite presence of a considerable wind.

The deterministic chaos in the atmospheric motions was also discovered on a simple model of free convection [2] (see for a recent review Ref. [3]). In present paper the deterministic chaos approach has been generalized on the case with randomly fluctuating parameters and takes the form of distributed chaos, that allows consideration of thermal convection with large Rayleigh (Reynolds) numbers typical for atmosphere. This consideration is based on the Bolgiano-Obukhov phenomenology [4- 7 ] and supported by comparison with direct numerical simulations, laboratory experiments and atmospheric measurements in a wide range of the spatio-temporal scales.

In Section II the free convection in the Boussinesq approximation and the Kolmogorov-Bolgiano-Obukhov approach for the inertial-buoyancy range have been considered both for unstable and stable stratification. In Section III the Bolgiano-Obukhov spatial distributed chaos has been introduced. In Section IV results of laboratory experiments for large Rayleigh numbers have been discussed and compared with the predictions made for the Bolgiano-Obukhov spatial distributed chaos. In Section $\mathrm{V}$ these predictions have been compared with results of measurements made in the atmospheric boundary layer (over sea and land) and with results of measurements in the Martian atmospheric boundary layer (using the data obtained with the Phoenix lander). In Sections VI-VIII the predictions have been compared with measurements in the troposphere, tropopause and stratosphere for the low-, mid- and high-latitudes bands. In Section IX this approach has been applied to the spatial temperature fluctuations in clouds. In Section X an analogous consideration for the Venus's, Mars's, Jupiter's and Saturn's atmospheres has been briefly discussed using the recent observational data.

\section{FREE THERMAL CONVECTION}

The buoyancy driven convection in the Boussinesq approximation is described by equations [8]

$$
\begin{gathered}
\frac{\partial \mathbf{u}}{\partial t}+(\mathbf{u} \cdot \nabla) \mathbf{u}=-\frac{\nabla p}{\rho_{0}}+\sigma g \theta \mathbf{e}_{z}+\nu \nabla^{2} \mathbf{u} \\
\frac{\partial \theta}{\partial t}+(\mathbf{u} \cdot \nabla) \theta=S \frac{\Delta}{H} e_{z} u_{z}+\kappa \nabla^{2} \theta \\
\nabla \cdot \mathbf{u}=\mathbf{0}
\end{gathered}
$$

where $\theta$ is the temperature fluctuations over a temperature profile $T_{0}(z), p$ is the pressure, $\mathbf{u}$ is the velocity, $\mathbf{e}_{z}$ is a unit vector along the gravity direction, $g$ is the gravity acceleration, $H$ is the distance between the layers and $\Delta$ is the temperature difference between the layers, $\rho_{0}$ is the mean density, $\nu$ is the viscosity and $\kappa$ is the thermal diffusivity, $\sigma$ is the thermal expansion coefficient. For the unstable stratification $S=+1$ and for the stable stratification $S=-1$.

These equations at $\nu=\kappa=0$ have an invariant

$$
\mathcal{E}=\int_{V}\left(\mathbf{u}^{2}-S \sigma g \frac{H}{\Delta} \theta^{2}\right) d \mathbf{r}
$$

where $V$ is the spatial volume (see, for instance, Ref. [8]). It can be readily shown that presence of a global rotation does not change the invariant Eq. (4). 


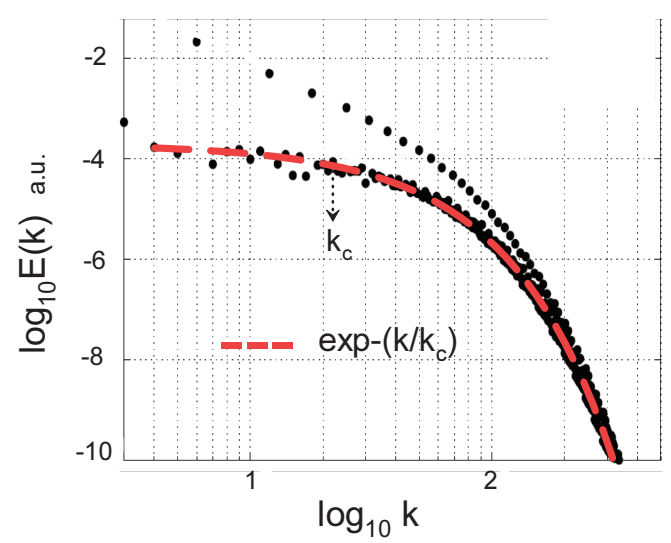

FIG. 1: Power spectrum of the temperature fluctuations at $\operatorname{Pr}=1$ and $R a=6.6 \times 10^{6}$ (onset of the convective turbulence).

Corresponding generalization of the KolmogorovBolgiano-Obukhov approach for the inertial-buoyancy range of the spatial scales [6] provides a relationship between characteristic temperature fluctuations $\theta_{c}$ and the characteristic wavenumber scale $k_{c}$

$$
\theta_{c} \propto(\sigma g)^{-1} \varepsilon^{2 / 3} k_{c}^{1 / 3}
$$

where the generalized dissipation/transfer rate

$$
\varepsilon=\left|\frac{d\left\langle\mathbf{u}^{2}-S \sigma g \frac{d}{\Delta} \theta^{2}\right\rangle}{d t}\right|
$$

$\langle\ldots\rangle$ denotes spatial averaging.

When the buoyancy forces dominate over the inertial forces (see for the stable stratification Ref. [6] and for the unstable one Refs. 9]-11]) one can use

$$
\varepsilon_{b}=\left|\frac{d\left\langle\theta^{2}\right\rangle}{d t}\right|
$$

instead of the $\varepsilon$ and then one obtains

$$
\theta_{c} \propto(\sigma g)^{-1 / 5} \varepsilon_{b}^{2 / 5} k_{c}^{1 / 5}
$$

instead of the relationship Eq. (5).

\section{SPATIAL DISTRIBUTED CHAOS}

For the bounded and smooth dynamical systems the exponential frequency spectrum

$$
E(f) \propto \exp \left(-f / f_{c}\right)
$$

is usually associated with deterministic chaos (see, for instance, Refs. 12-16 and Section VI).

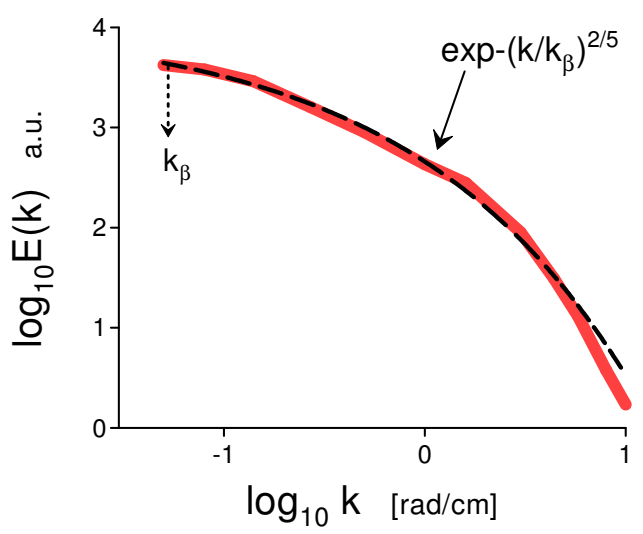

FIG. 2: Horizontal power spectrum of the experimentally observed temperature fluctuations near the lower boundary at $R a \sim 10^{11}$

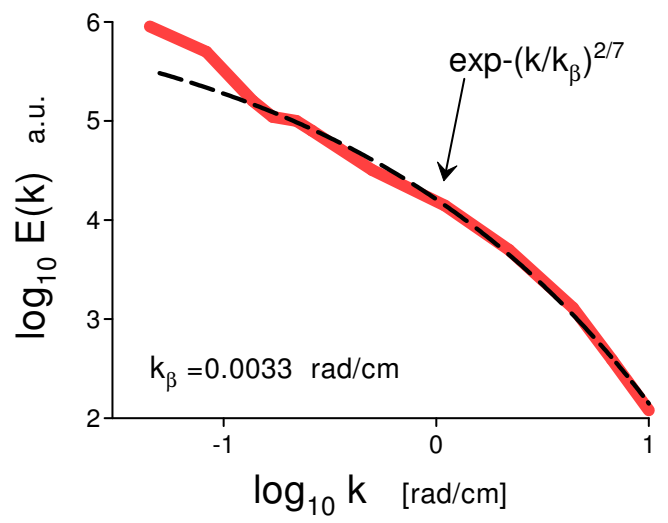

FIG. 3: The same as in Fig. 2 but near the middle of the mixed layer.

For the spatial (wavenumber) domain this spectrum is replaced by the spectrum

$$
E(k) \propto \exp \left(-k / k_{c}\right)
$$

(see, for instance, Ref. [12] and references therein).

In the buoyancy driven unstably stratified thermal convection at the Prandtl number $\operatorname{Pr}=\nu / \kappa \sim 1$ transition to turbulence occurs at the Rayleigh number $R a \sim 10^{6}$ 17. Figure 1 shows (in the log-log scales) power spectrum of temperature fluctuations at $\operatorname{Pr}=1$ and $R a=6.6 \times 10^{6}$ obtained in a direct numerical simulation reported in Ref. 18 (the spectral data were taken from the Fig. 11 of the Ref. [18). Two branches of this spectrum correspond to different number of the Fourier modes: the upper branch to a small number whereas the lower branch corresponds to the most of 
the modes. Therefore, the only lower one has a physical significance. The dashed curve in the Fig. 1 indicates the exponential spectrum Eq. (10). The dotted arrow indicates position of the $k_{c}$.

Transition to turbulence usually results in fluctuations of the parameter $k_{c}$. These fluctuations can be taken into account by an ensemble averaging

$$
E(k) \propto \int_{0}^{\infty} P\left(k_{c}\right) \exp -\left(k / k_{c}\right) d k_{c} \propto \exp -\left(k / k_{\beta}\right)^{\beta}
$$

The stretched exponential in the right-hand side of the Eq. (11) is generalization of the exponential spectrum Eq. (10). An estimation of the asymptotic (at large $k_{c}$ ) behaviour of the probability distribution $P\left(k_{c}\right)$

$$
P\left(k_{c}\right) \propto k_{c}^{-1+\beta /[2(1-\beta)]} \exp \left(-\gamma k_{c}^{\beta /(1-\beta)}\right)
$$

(where $\gamma$ is a constant) can be made from the Eq. (11) [19].

The Eqs. (5) and (8) can be considered in a general form

$$
\theta_{c} \propto k_{c}^{\alpha}
$$

When $\theta_{c}$ has a Gaussian distribution 6] relationship between parameters $\alpha$ and $\beta$

$$
\beta=\frac{2 \alpha}{1+2 \alpha}
$$

follows immediately from the Eqs. (12-13).

For the Eq. (5) $\alpha=1 / 3$, hence $\beta=2 / 5$ and

$$
E(k) \propto \exp -\left(k / k_{\beta}\right)^{2 / 5} .
$$

For the Eq. (8) $\alpha=1 / 5$, hence $\beta=2 / 7$ and

$$
E(k) \propto \exp -\left(k / k_{\beta}\right)^{2 / 7},
$$

Generally the parameter $k_{\beta}$ has no direct geometrical meaning, but sometimes it can be related to spatial scales of the dominating coherent structures.

\section{LABORATORY EXPERIMENTS}

In the paper Ref. 20] results of a laboratory simulation of atmospheric free (unstable stratification) convection were reported. The experiment was performed in water at Rayleigh number $R a \sim 10^{11}$. The convection chamber horizontal dimensions were $122 \times$ $144 \mathrm{~cm}$ with the depth $76 \mathrm{~cm}$.

Figure 2 shows a horizontal spatial power spectrum of the experimentally observed temperature fluctuations near the lower boundary (the spectral data were taken

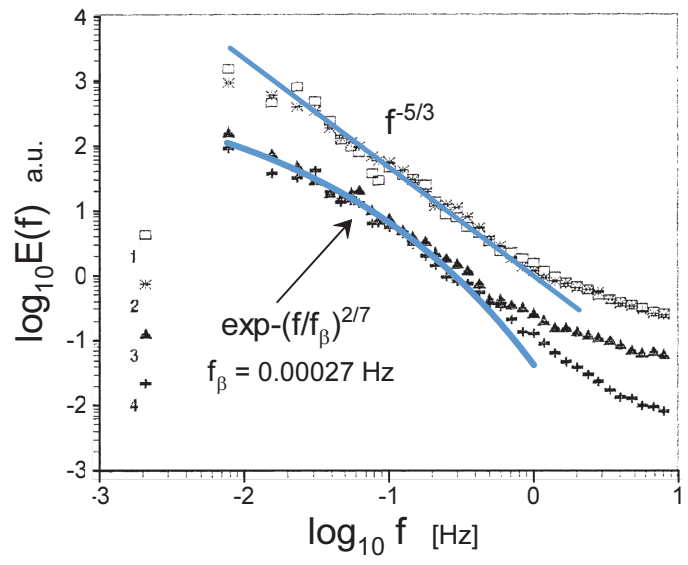

FIG. 4: Four power spectra of the temperature fluctuations in the atmospheric surface layer over sea at near free convection conditions.

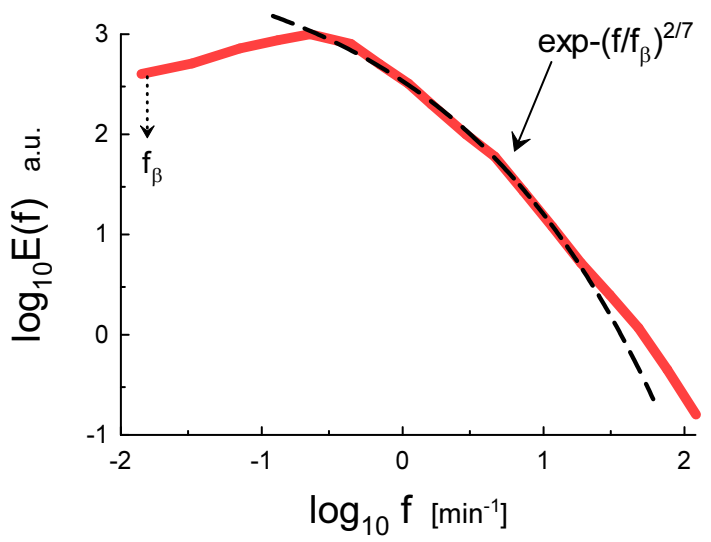

FIG. 5: Power spectra of the temperature fluctuations in the atmospheric surface layer over land at near free convection conditions.

from Fig. 13a of the Ref. [20]). The dashed curve in the Fig. 2 indicates correspondence to the stretched exponential Eq. (15).

Figure 3 shows a horizontal spatial power spectrum of the experimentally observed temperature fluctuations near the middle of the mixed layer (the spectral data were taken from Fig. 13b of the Ref. 20]). The dashed curve in the Fig. 3 indicates correspondence to the stretched exponential Eq. (16). 


\section{ATMOSPHERIC BOUNDARY LAYER}

\section{A. Free convection}

The Ref. 24] reports results of measurements of the temperature fluctuations in the near free convection (at unstable stratification) by a fixed probe over sea surface (the height of the tower was $\sim 12 \mathrm{~m}$ above the sea surface). The weather was calm and the sea surface was aerodynamically smooth. In the air the r.m.s. horizontal velocity fluctuations were comparable to the wind gusts. Results for four data sets obtained during a day were reported: two with comparatively high temperature fluctuations and two with low ones. Figure 4 shows the four power spectra of the temperature fluctuations corresponding to the four data sets (the spectral data were taken from Fig. 1c of the Ref. [24]). These are frequency spectra. However, in the case of free convection the mean flow velocity $U_{0}$, usually used for the Taylor hypothesis [6], [12] relating $f$ and $k: f=U_{0} k / 2 \pi$, can be replaced by a characteristic velocity of advection by the energy-containing eddies past the probe [21, 22 ] (see also Ref. 23 and references therein) and we should compare the observed frequency spectra with the spatial (wavenumber) spectral prediction.

The solid straight line is drawn to indicate the " $-5 / 3$ " power law for the strong fluctuations case [6], whereas the curved solid line is drawn to indicate the stretched exponential Eq. (16) for the weak fluctuations case.

The Ref. 25] reports results of measurements of the temperature fluctuations in the near free convection over land. The measurements were produced by 'Eddycovariance' tower (at $2.29 \mathrm{~m}$ height) and Sodar/RASS system in the morning hours (from 07:35 to 08:40 UTC) over a corn field. Moderate to high buoyancy fluxes and a drop of the wind speed result in domination of buoyancy over shear. The occurrence of large-scale turbulence structures (plumes) were registered. The free convection conditions occur at about 50 percent of the 92 days of the measurement period. Figure 5 shows power spectrum of the temperature fluctuations (the sonic spectral data were taken from Fig. $6 \mathrm{~h}$ of the Ref. [25]). The dashed line is drawn to indicate the stretched exponential Eq. (16) and the dotted arrow indicates position of $f_{\beta}$.

\section{B. Non-free convection}

It is well known (although not well understood) that the free-like convection behaviour can be often observed under conditions which seem to be rather different from the free ones.

Figure 6, for instance, shows power spectrum of the temperature fluctuations airborne measured in the

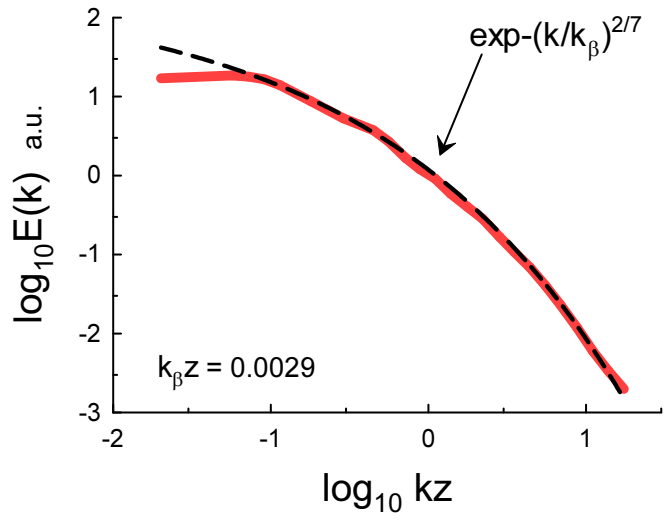

FIG. 6: Power spectra of the alongwind-sampled temperature fluctuations in the unstable atmospheric surface layer over ocean.

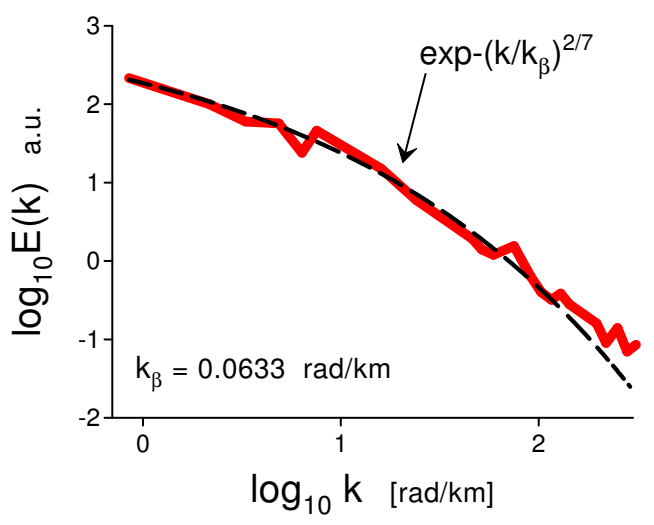

FIG. 7: Power spectra of the alongwind-sampled temperature fluctuations over a flat and uniform land terrain with a large upward heat flux.

Weastern Atlantic ocean at $50 \mathrm{~m}$ level above the water surface during cold air outbreaks [26]. The measurements were made in near-shore areas of roll vortices and cloud streets under considerable mean wind shear. The alongwind-sampled spectral data were taken from Fig. 4 of the Ref. [26] ( $k$ is the alongwind wavenumber, $z$ is the height above the ocean surface). The wavenumber was calculated using the Taylor hypothesis $k=2 \pi f / V_{a}$, where $V_{a}$ is the relative velocity between the aircraft and air. The dashed line is drawn to indicate the stretched exponential Eq. (16).

Figure 7 shows power spectrum of the temperature fluctuations airborne measured in Eastern Colorado at $100 \mathrm{~m}$ level above a flat, uniform and treeless land terrain with a large upward heat flux (the alongwind-sampled 


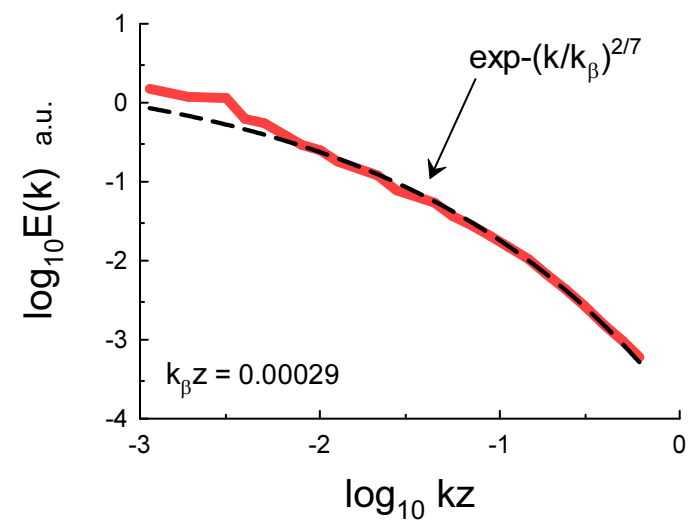

FIG. 8: Power spectrum of the alongwind temperature fluctuations observed in the Martian atmospheric boundary layer.

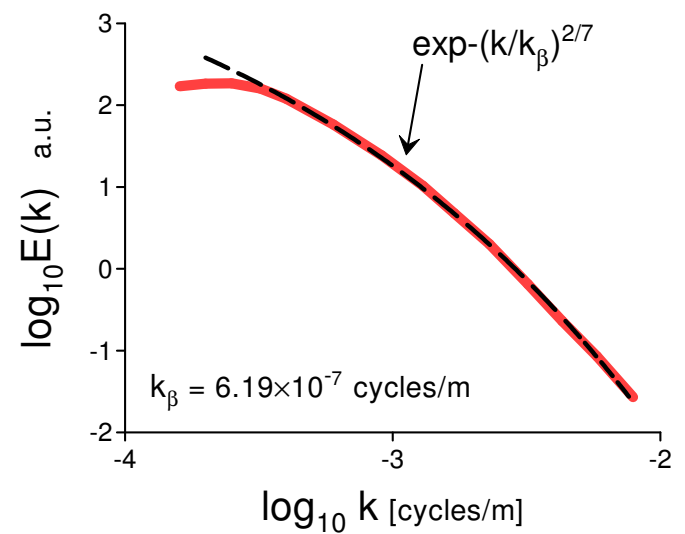

FIG. 9: Vertical wavenumber power spectra of temperature fluctuations for the troposphere: the low-latitude band.

spectral data were taken from Fig. 13 of the Ref. 27)). The heat transporting eddies (thermals) were elongated along the wind (the mean wind speed was $\sim 10 \mathrm{~m} / \mathrm{sec}$ ). A cold front had passed over the area several ours earlier.

\section{Martian atmospheric boundary layer}

Main physical properties of the 'climate and meteorology' related to the Martian atmosphere turned out to be surprisingly similar to those of the Earth atmosphere (see for a review Refs. 28, 291) and references therein). Although the Martian atmosphere (consisting mostly of $\mathrm{CO}_{2}$ ) is thinner than that of Earth the thermal (buoyancy driven) convection processes in this atmosphere are no less vigorous and substantial than those in the Earth atmosphere. The main difference in this respect is the strong influence of water (in different phases) on the Earth's atmospheric dynamics, whereas the dust is

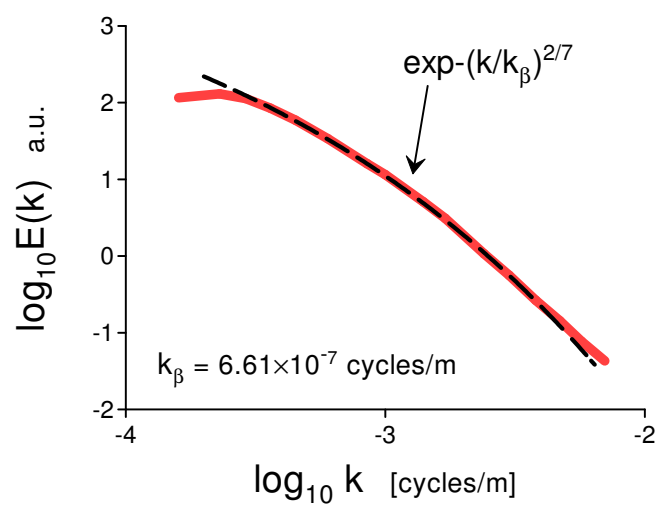

FIG. 10: As in the Fig. 9 but for the mid-latitude band.

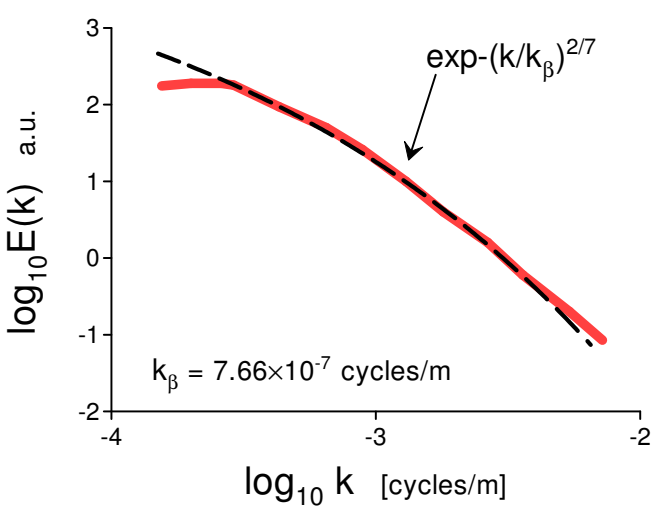

FIG. 11: As in the Fig. 9 but for the high-latitude site (Davis, Antarctica).

the rather significant factor in the Martian atmospheric dynamics. Therefore it is interesting to compare the above results obtained for Earth with those available now for Mars .

We have already seen in the Earth atmospheric boundary layer (Figs. 6 and 7) that the free-like convection behaviour can be observed under conditions which seem to be rather different from the free ones. Figure 15 shows power spectrum of the alongwind temperature fluctuations observed in the Martian atmospheric boundary layer by the Phoenix lander (the spectral data were taken from Fig. 8 of the Ref. [30]). The measurements were made at about $2 \mathrm{~m}$ height above the Mars surface ( $k$ is the alongwind wavenumber, $z$ is the height above the surface, see the Ref 30 for more details). As for the Fig. 6 the wavenumber was calculated using the Taylor hypothesis $k=2 \pi f / V$, where $V$ is the mean wind speed. The dashed line is drawn to indicate the 


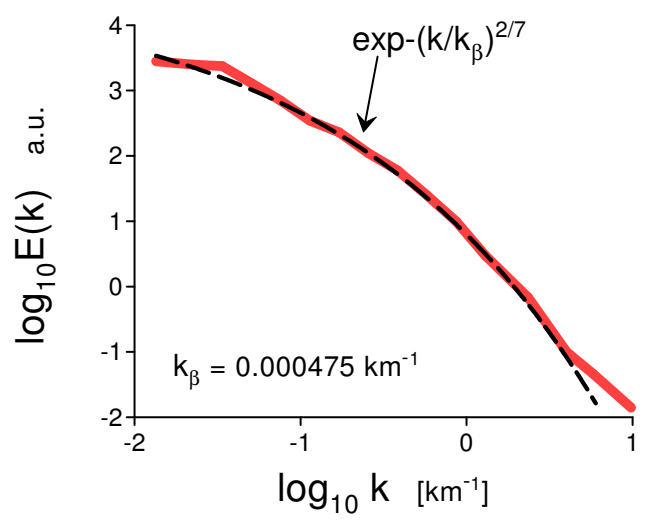

FIG. 12: Horizontal power spectra of the temperature fluctuations in the lowermost stratosphere (near the tropopause) over the South Pacific, for high dissipation rates.

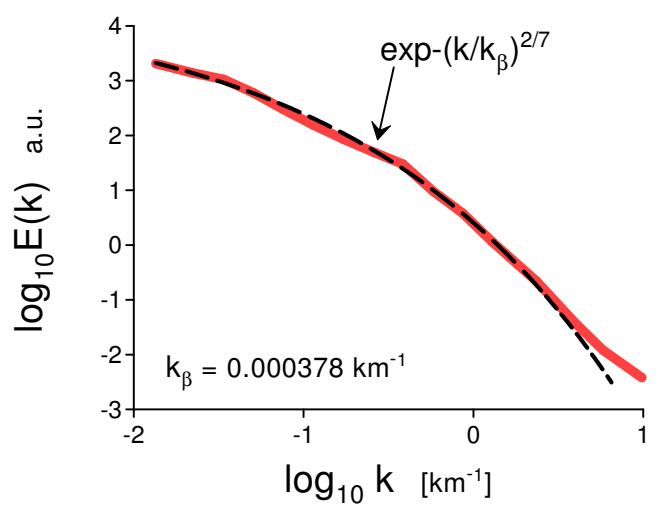

FIG. 13: As in the Fig. 12 but for small dissipation rates.

stretched exponential Eq. (16).

\section{TROPOSPHERE}

Systematic studies of the tropospheric and stratospheric temperature variability were made by the Australian Bureau of Meteorology over Australia and Antarctica using high-resolution radiosonde measurements . These measurements cover a longitude range of $78^{\circ} \mathrm{E}-159^{\circ} \mathrm{E}$ and a latitude range of $12^{\circ} \mathrm{S}-68^{\circ} \mathrm{S}$ and were performed for the time period 1990-1993 years. In the paper Ref. 31 results of a spectral analysis of the

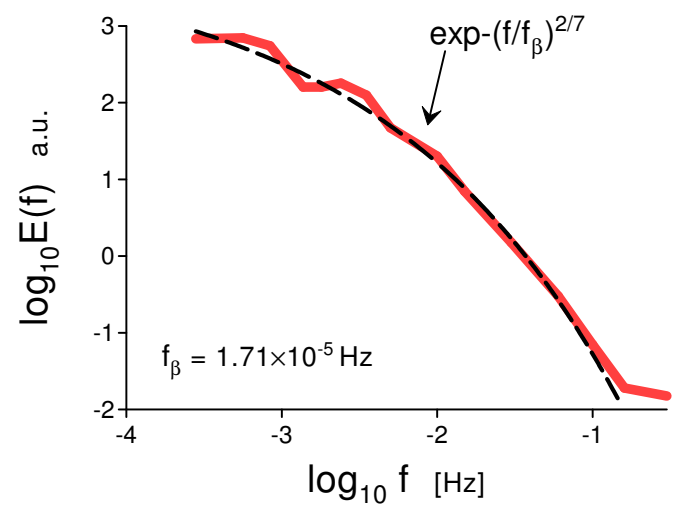

FIG. 14: Horizontal power spectra of the temperature fluctuations in tropopause over North Atlantic.

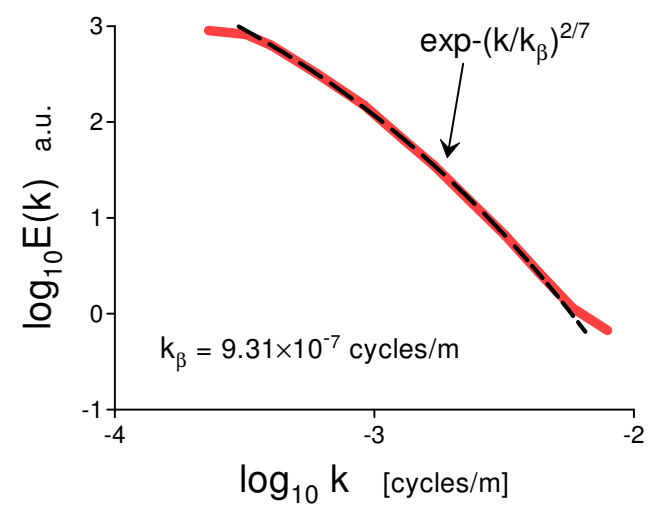

FIG. 15: As in Fig. 9 but for stratosphere.

data were presented and we have chosen three latitude bands: Gove, Darwin (low latitudes), Wagga, Albany, Adelaide (mid-latitudes), Davis (high-latitude site, Antarctica) . The tropopause over Darwin is located near $16 \mathrm{~km}$ whereas over Davis it is near $9 \mathrm{~km}$.

Figures 9-11 show the vertical wavenumber power spectra of temperature fluctuations for the troposphere: the low, middle and high latitudes, respectively (the spectral data were taken from Fig. 6 of the Ref. [31). The dashed curve is drawn to indicate the stretched exponential Eq. (16). 


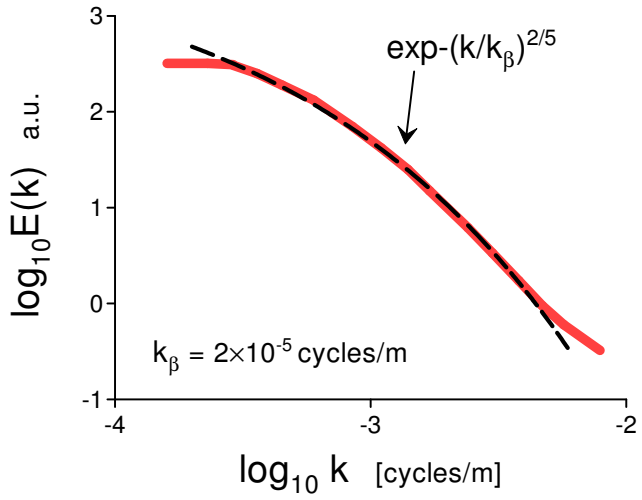

FIG. 16: As in Fig. 10 but for stratosphere.

\section{TROPOPAUSE}

It is known that the stratification is especially strong in the tropopause inversion layer (see, for instance Ref. 32 and references therein). In a recent paper Ref. 33] a spectral analysis of the data obtained during the long-distance flights over the South Pacific near New Zealand (the DEEPWAVE experiment 34) was performed. The measurements were made in the lowermost stratosphere. Figures 12 and 13 shows the horizontal power spectra of the temperature fluctuations for high and small dissipation rates respectively (the spectral data were taken from Fig.4b of the Ref 33]). The dashed curve is drawn to indicate the stretched exponential Eq. (16).

In another recent paper Ref. 35. results of an analogous experiment over the North Atlantic were reported. The measurements were made in the tropopause or slightly above the tropopause region. Figure 14 shows the horizontal power spectra of the temperature fluctuations (the spectral data were taken from Fig. 6b of the Ref. 35. The dashed curve is drawn to indicate the stretched exponential Eq. (16) (the Taylor hypothesis can be used for transformation of the frequency spectrum into a wavenumber one). It is interesting to note that the data shown in the Fig. 14 were obtained for the time period 18.19 - 19.18 UTC, whereas for the time period 14.20 -15.00 UTC the spectral data shows clear indication of the scaling $k^{-5 / 3}$ spectrum (cf. Fig. 6a in the Ref. 35 and Fig. 4 in present paper).

\section{STRATOSPHERE}

Figure 15 shows the vertical wavenumber power spectra of temperature fluctuations for the stratosphere at the low latitudes band (Gove, Darwin). The spectral

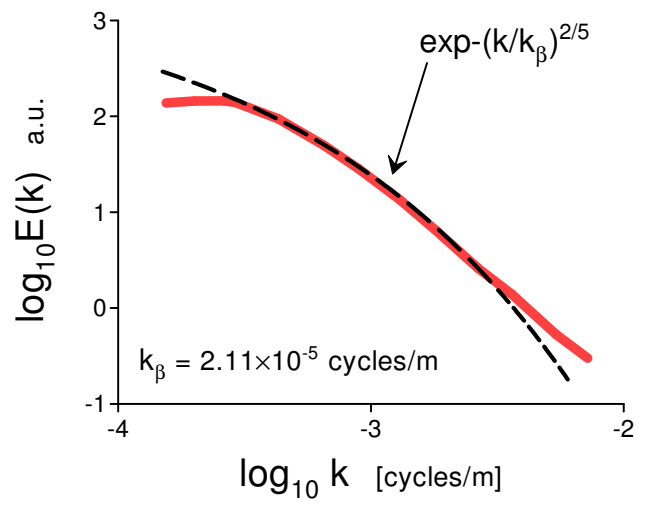

FIG. 17: As in Fig. 11 but for stratosphere.

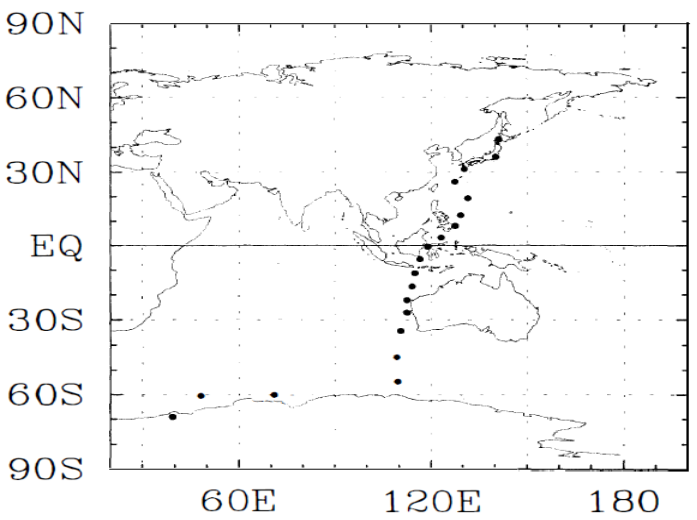

FIG. 18: The observation points along the vessel cruise (the routine ozonesonde observational points are also added).

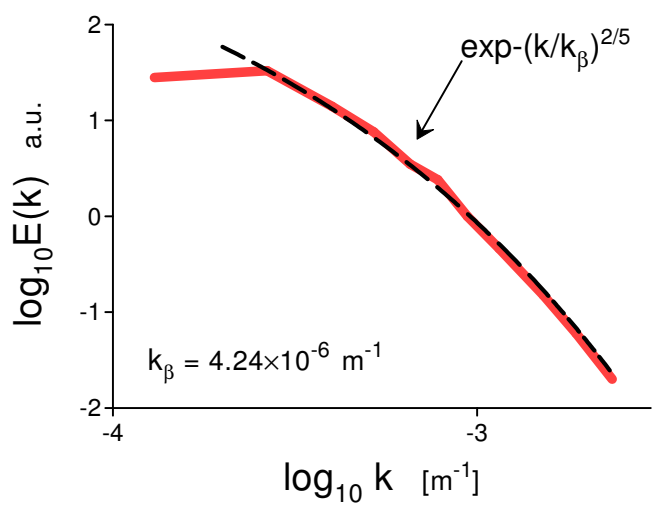

FIG. 19: Vertical power spectra of the temperature fluctuations in the lower stratosphere. 


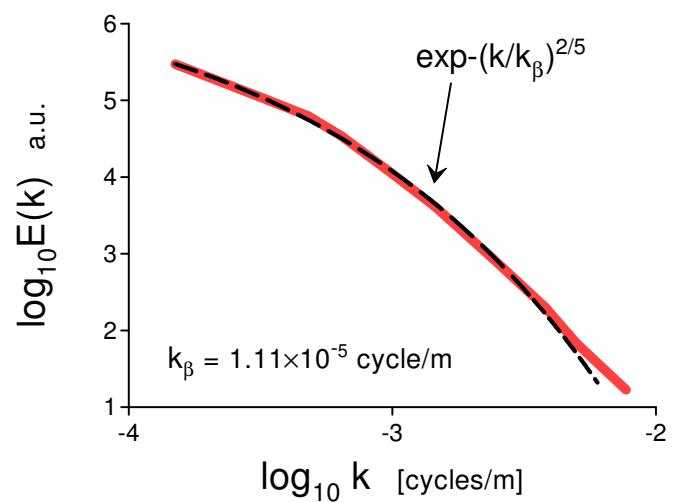

FIG. 20: Vertical power spectrum of the temperature fluctuations in the winter troposphere (over Illinois).

data were taken from the Fig. 6 of the Ref. 31] (cf. Fig. 9 of the present paper). The dashed curve is drawn to indicate the stretched exponential Eq. (16). Figure 16 shows the vertical wavenumber power spectra of temperature fluctuations for the stratosphere at the mid-latitudes latitudes band (Wagga, Albany, Adelaide) and Fig. 17 shows the vertical wavenumber power spectra of temperature fluctuations for the stratosphere at the high-latitudes latitudes band (Davis, Antarctica). The spectral data for the Figs 16 and 17 were taken from the Fig. 6 of the Ref. 31 (cf. Fig. 10 and 11 of the present paper). The dashed curves in the Figs. 16 and 17 are drawn to indicate the stretched exponential Eq. (15). One can see that, unlike for the troposphere, for the stratosphere the spectral law is changing from the Eq. (16) into Eq. (15) at the mid- and high-latitudes.

In paper Ref. 36 results of the 4 year ozonesonde observations in the lower stratosphere were reported. Measurements were made over a latitudinal region $60^{\circ} \mathrm{S}-43^{\circ} \mathrm{N}$ with a research oceanic vessel as a base. Figure 18 shows the observation points along the vessel cruise in 1988 year (November and December). The additional points correspond to the routine ozonesonde stations at Naha, Kagoshima, Tsukuba and Sapporo. Vertical distribution of temperature in the altitude range $19-27 \mathrm{~km}$ were measured.

Figure 19 shows vertical wavenumber power spectra of the temperature fluctuations (averaged over all observations). The spectral data were taken from Fig. 5 of the Ref. 36. The dashed curve is drawn to indicate the stretched exponential Eq. (15).

The spectral law Eq. (15) was also observed in the troposphere. Figure 20, for instance, shows vertical power spectrum of the temperature fluctuations observed in the winter troposphere over the very flat terrain in central Illinois using the several hundred balloons. The spectral

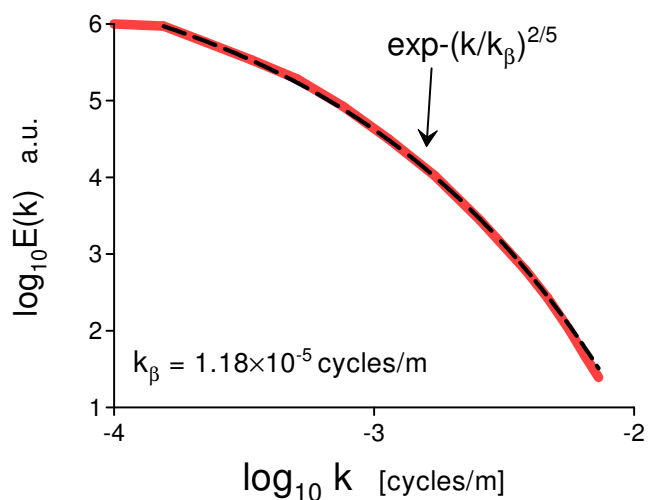

FIG. 21: As in Fig. 20 but in the lower stratosphere.

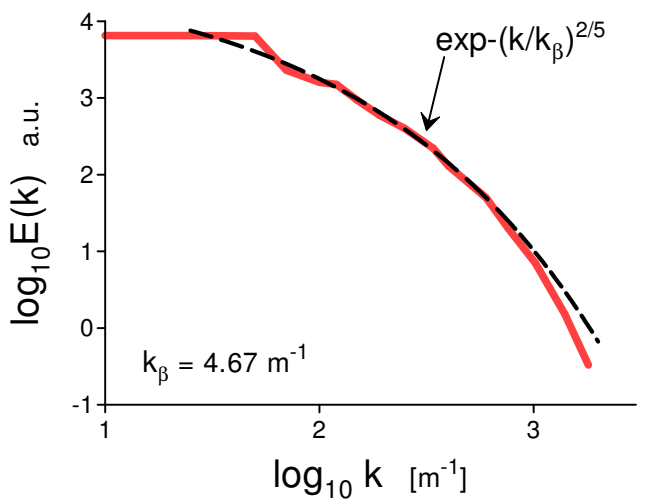

FIG. 22: Horizontal power spectra of the temperature fluctuations at the cloud top interface for the dimensionless time $t=3$.

data were taken from Fig. 3 of the Ref. 37]. Figure 21 shows, for comparison, the power spectrum obtained at the same conditions but in the lower stratosphere. The spectral data were also taken from the Fig. 3 of the Ref. 37 .

\section{CLOUD TOP INTERFACE}

The estimate Eq. (5) and, consequently, the spectrum Eq. (15) can be also applied to a certain range of scales and to certain areas of the clouds where the buoyancyinertial effects dominate over other physical effects. In this sense the cloud top interface is of especial interest. In recent paper Ref. 38 a direct numerical simulation of mixing through an atmospheric warm cloud and clear air interface, including collision and coalescence of the droplets, was performed using a Boussinesq-like approximation. The transient evolution of the cloud top interface was studied with an initial mono-disperse 


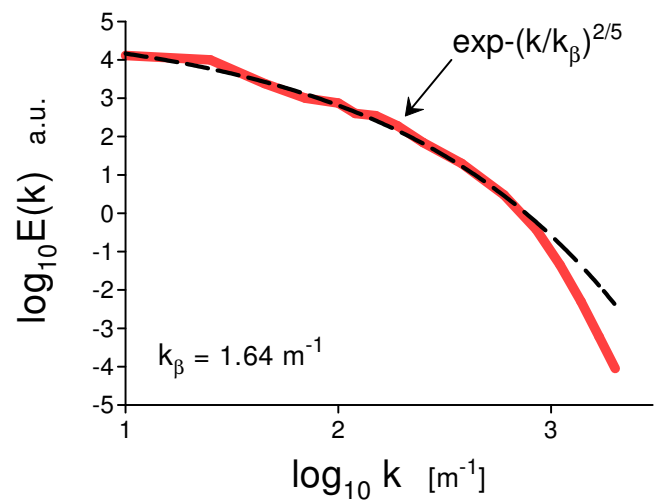

FIG. 23: As in Fig. 22 but for $t=18$.

cloud droplet population. Initial Taylor-Reynolds number of cloud and air were taken as $R e_{\lambda}=90$ and 20 respectively. An unstably stratified temperature profile was used in this simulation, and on the horizontal plane the initial water vapour and temperature fields were uniform. The buoyancy force was generated by the variations of water vapour density and temperature. The initial eddy turnover time was used in order to obtain a dimensionless time for this simulation.

Figures 22 and 23 show horizontal power spectra of the temperature fluctuations obtained at the top interface for the dimensionless times $t=3$ and $t=18$ respectively (the spectral data were taken from Fig. $5 \mathrm{f}$ of the Ref. [38). The dashed curves are drawn to indicate the stretched exponential Eq. (15).

Already first NASA geosynchronous meteorological satellite (SMS-1) provided the thermal infrared (10.5$12.6 \mu \mathrm{m}$ ) images which allowed estimate the cloud brightness on the global scales. For these images the lower temperatures correspond to higher intensity levels. Therefore, for these images the colder tops of the clouds are the dominant feature. Figures 24 and 25 show power spectra of the fluctuations of the cloud brightness vs. planetary wavenumber $k$ for a mid-latitude zone $\left(25-60^{\circ} \mathrm{N}\right)$ and for an equatorial region $\left(15^{\circ} \mathrm{N}-15^{\circ} \mathrm{S}\right)$, respectively . The spectral data were taken from Fig. 4 of the Ref. 39. The dashed curves are drawn to indicate the stretched exponential Eq. (15).

\section{ATMOSPHERE OF VENUS, MARS, JUPITER AND SATURN}

Although the Venus's, Mars's, Jupiter's and Saturn's atmospheres are rather different from that on Earth it seems that the thermal convection also plays very significant role in their atmospheric dynamics. Moreover, as we will see below, the same spectral

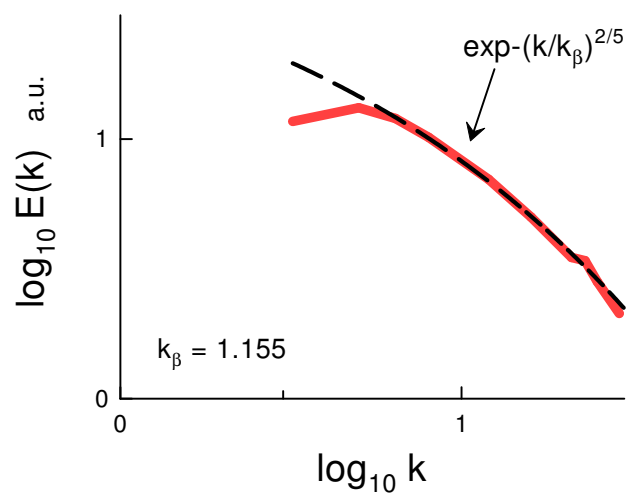

FIG. 24: Cloud thermal infrared brightness fluctuations power spectrum at the mid-latitudes zone $\left(25-60^{\circ} \mathrm{N}\right)$.

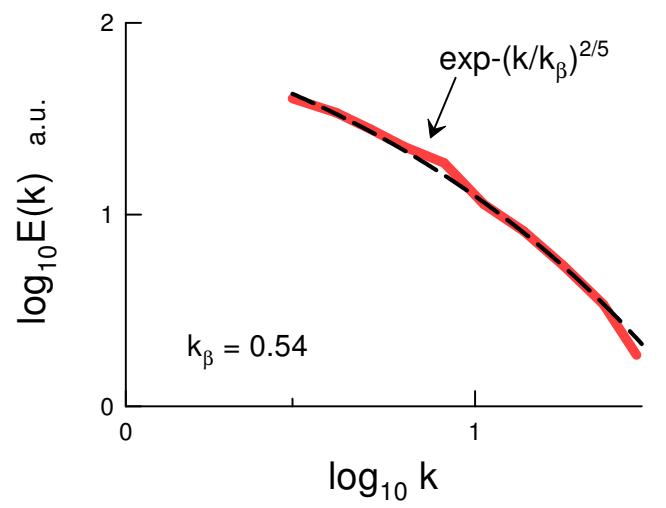

FIG. 25: As in Fig. 24 but for the equatorial region $\left(15^{\circ} \mathrm{N}-\right.$ $\left.15^{\circ} \mathrm{S}\right)$.

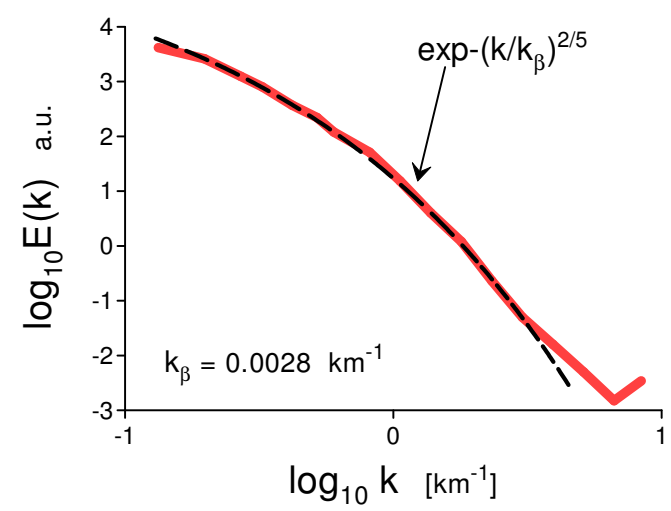

FIG. 26: Vertical power spectrum of the temperature fluctuations in the Venus's atmosphere. 


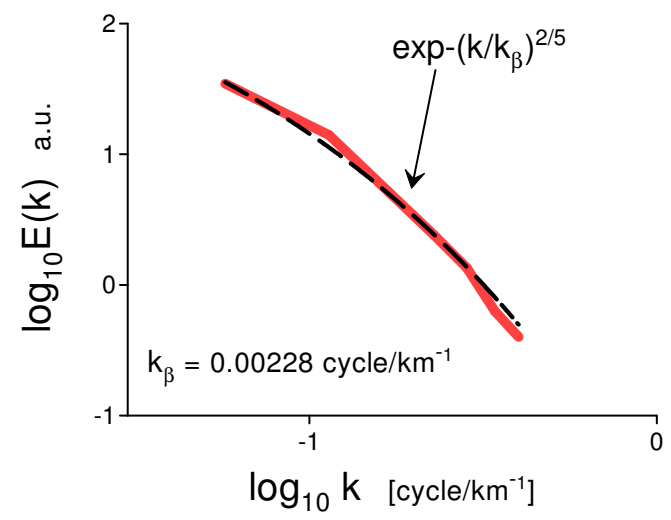

FIG. 27: Vertical power spectrum of the temperature fluctuations in the Mars's atmosphere in the altitude range of $3-20 \mathrm{~km}$.

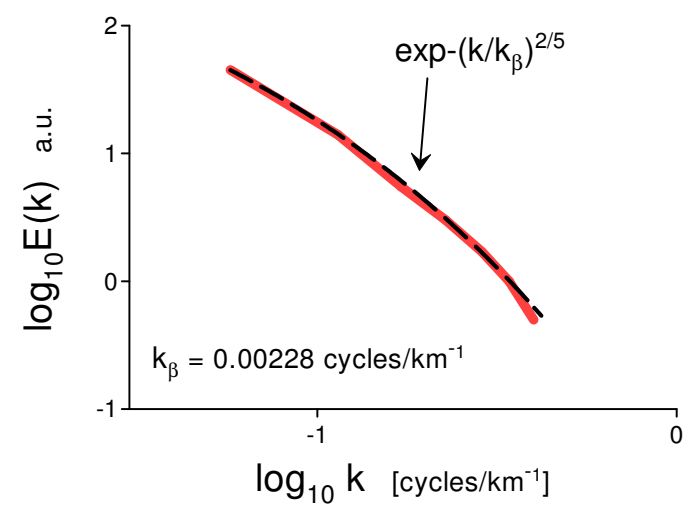

FIG. 28: As in Fig. 27 but in the altitude range of $15-32 \mathrm{~km}$.

law Eq. (15) can be applied to these atmospheres as well.

In paper Ref. [40] the vertical wavenumber spectra of temperature fluctuations obtained for the Venus's atmosphere were reported. The data were obtained during the Venus Express mission for the period from July 2006 to June 2010 by the radio occultation method.

Figure 26 shows a vertical temperature power spectrum averaged over the latitude bands $90-75^{\circ} \mathrm{S}$ and $75-90^{\circ} \mathrm{N}$ in the altitude range of $65-80 \mathrm{~km}$ (the spectral data were taken from Fig. 3 of the Ref. 40). The dashed curve is drawn to indicate the stretched exponential Eq. (15).

In paper Ref. 41 the vertical wavenumber spectra of temperature fluctuations obtained for the Mars's atmosphere were reported. The data were obtained during the Mars Global Surveyor radio occultation experiments from January 1998 to September 2006. A

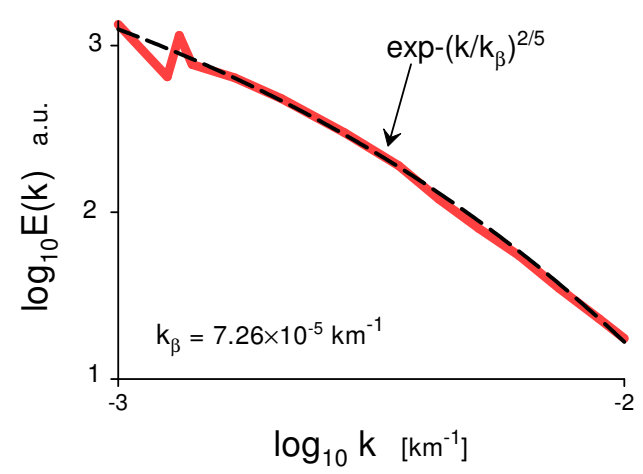

FIG. 29: Power spectrum for the mid-infrared thermal emission for Jupiter's south polar atmosphere ( $k$ is the horizontal wavenumber).

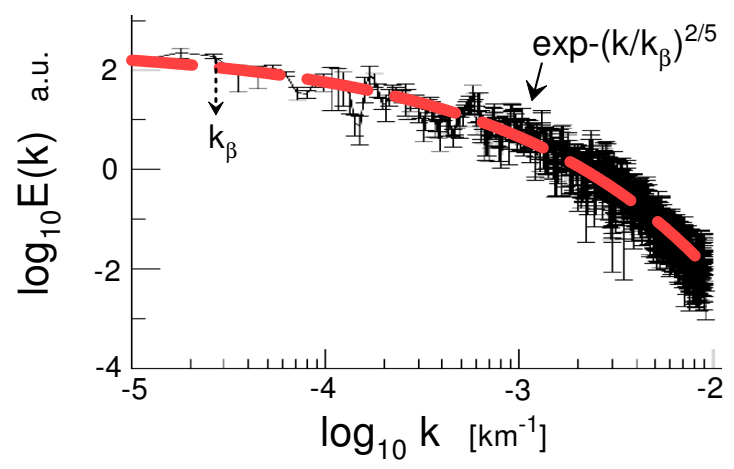

FIG. 30: As in the Fig. 18 but for a narrow circular path outside of the cyclones' belt near Jupiter's South Pole.

3.6-cm wavelength radio signal was transmitted by the Mars Global Surveyor and received on Earth.

Figures 27 and 28 show a vertical temperature power spectrum averaged over the latitude band 75$45^{\circ} \mathrm{S}$ in the altitude ranges of $3-20 \mathrm{~km}$ (the spectral data were taken from Fig. 3a of the Ref. 41]) and of $15-32 \mathrm{~km}$ (the spectral data were taken from Fig. 4a of the Ref. 41]), respectively. The dashed curves are drawn to indicate the stretched exponential Eq. (15).

The recent papers Refs. 42, 43, reported an analysis of the data obtained with the Jovian Infrared Auroral Mapper (JIRAM) onboard the Juno mission for the Jupiter's South Pole atmosphere. Figure 29 shows an example of the power spectra for the mid-infrared (thermal) emission for Jupiter's south polar atmosphere (the latitudes higher than $82^{\circ} \mathrm{S}$ ). This region was dominated by the polar cyclones. The cyclones are characterized by 


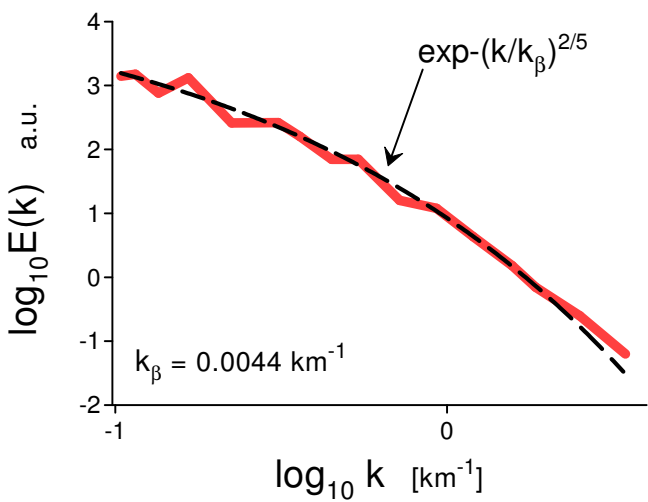

FIG. 31: Power spectrum of the stratospheric temperature fluctuations for Saturn ( $k$ is the vertical wavenumber).

thick clouds. At the presence of a thick cloud cover the JIRAM is sensible to the temperatures of the cloud top. The spectral data for the Fig. 29 were taken from the Fig. 11c (the upper curve) of the Ref. [42. The dashed line in the Fig. 29 indicates the stretched exponential decay Eq. (15).

Figure 30 shows power spectrum for the mid-infrared (thermal) emission obtained with the JIRAM for a narrow circular path outside of the cyclones' belt near Jupiter's South Pole. For this region the cloud cover is thinner than that for the region dominated by the polar cyclones and the winds have low speed. The spectral data were taken from Fig. 2 of the Ref. [43. The dashed line in the Fig. 30 indicates the stretched exponential decay Eq. (15).

In the Ref. 44 a power spectrum of the temperature fluctuations in Saturn's stratosphere was reported. The temperature fluctuations were obtained for the vertical temperature profile at $55.5^{\circ} \mathrm{S}$ latitude using the NASA Infrared Telescope on Mauna Kea. The inverted occultation method was used for this purpose. Figure 31 shows the power spectrum (the spectral data were taken from Fig. $7 \mathrm{~b}$ of the Ref. 44]). The dashed line in the Fig. 31 indicates the stretched exponential decay Eq. (15).

\section{CONCLUSIONS}

The Bolgiano-Obukhov phenomenology appears to be adequate for the atmospheric free convection in the atmospheric boundary layer (both over land and sea) when it is considered in the frames of the distributed chaos approach. Moreover, it can be also applied to the atmospheric data for the temperature fluctuations even in some cases when the free convection conditions are not satisfied and for the planetary scales when the winds can be considered as a part of the planetary-scale thermal convection. The Martian atmospheric boundary layer and the higher levels of the Venus's, Martian's, Jupiter's and Saturn's atmosphere can be analyzed in the same way.
[1] T. Foken, Micrometeorology (Springer, BerlinHeidelberg, 2008)

[2] E.N. Lorenz, J. Atmos. Sci., 20, 130 (1963)

[3] S. Vannitsem, Chaos, 27, 032101 (2017)

[4] R. Bolgiano, J. Geophys. Res., 64, 2226 (1959)

[5] A.M. Obukhov, Dokl. Akad. Nauk SSSR, 125, 1246 (1959)

[6] A. S. Monin, A. M. Yaglom, Statistical Fluid Mechanics, Vol. II: Mechanics of Turbulence (Dover Pub. NY, 2007)

[7] E.S.C. Ching, Statistics and Scaling in Turbulent Rayleigh-Bénard Convection (Singapore: Springer, 2014)

[8] A. Kumar, A.G. Chatterjee and M.K. Verma, Phys. Rev. E, 90, 023016 (2014)

[9] I. Procaccia and R. Zeitak, Phys. Rev. Lett., 62, 2128 (1989)

[10] V.S. L'vov, Phys. Rev. Lett., 67, 687 (1991)

[11] G. Falkovich and V.S. L'vov, Physica D, 57, 85 (1992)

[12] J. E. Maggs and G. J. Morales, Phys. Rev. Lett., 107, 185003 (2011); Phys. Rev. E 86, 015401(R) (2012); Plasma Phys. Control. Fusion, 54, 124041 (2012)

[13] N. Ohtomo, K. Tokiwano, Y. Tanaka et. al., J. Phys. Soc. Jpn., 64, 1104 (1995)

[14] D.E. Sigeti, Phys. Rev. E, 52, 2443 (1995)

[15] J. D. Farmer, Physica D, 4, 366 (1982).

[16] U. Frisch and R. Morf, Phys. Rev., 23, 2673 (1981)
[17] M.K. Verma, Scholarpedia, 14, 53051 (2019)

[18] P.K. Mishra and M.K. Verma, Phys. Rev. E, 81, 056316 (2010)

[19] D.C. Johnston, Phys. Rev. B, 74, 184430 (2006)

[20] G.E. Willis and J.W. Deardorff, J. Atmos. Sci., 31, 1297 (1974)

[21] H. Tennekes, J. Fluid Mech., 67, 561 (1975)

[22] A. Kumar and M.K. Verma, R. Soc. open sci., 5, 172152 (2018)

[23] A. Bershadskii, Chaos 20, 043124 (2010)

[24] A.A. Grachev, Boundary-Layer Meteorology, 69, 27 (1994)

[25] R. Eigenmann, S. Metzger, and T. Foken, Atmos. Chem. Phys., 9, 8587 (2009)

[26] S.H. Chou and E.N. Yen, J. Atmos. Sci., 44, 3721 (1987)

[27] D.H. Lenschow. J. Appl. Meteorology, 9, 874 (1970)

[28] P.L. Read, S.R. Lewis and D.P. Mulholland, Rep. Prog. Phys., 78, 125901 (2015)

[29] W. Chen, S. Lovejoy, and J.-P. Muller, J. Geophys. Res. Atmos., 121, 11968 (2016)

[30] R. Davy, J.A. Davis, P.A. Taylor, C.F. Lange, W. Weng, J. Whiteway, and H.P. Gunnlaugson, J. Geophys. Res., 115, E00E13 (2010)

[31] S.J. Allen and R.A. Vincent, J. Geophys. Res., 100, 1327 (1995) 
[32] S. Gisinger, A. Dornbrack, V. Matthias et al., Monthly Weather Review, 145, 4249 (2017)

[33] U. Schumann, J. Atmos. Sci., 76, 3847 (2019)

[34] D.C. Fritts, R.B. Smith, M.J. Taylor et al., Bull. Amer. Meteor. Soc., 97, 425 (2016)

[35] D. Kunkel, P. Hoor, T. Kaluza et al., Atmos. Chem. Phys., 19, 12607 (2019)

[36] S.-Ya Ogino, M.D. Yamanaka, S. Kaneto, T. Yamanouchi and S. Fukao, Proc. NIPR Symp. Polar Meteorol. Glaciol., 11, 199 (1997)

[37] G. D. Nastrom and T.E. VanZandt, J. Geophys. Res., 106, D13, 14369 (2001)

[38] T. Bhowmick and M. Iovieno, Fluids, 4, 144 (2019)

[39] L.D. Travis, J. Atmos. Sci., 35, 1854 (1978)

[40] H. Ando, T. Imamura, T. Tsuda, S. Tellmann, M. Pat- zold, and B. Hausler, J. Atmos. Sci., 72, 2318 (2015)

[41] H. Ando, T. Imamura, T. Tsuda, J. Atmos. Sci., 69, 2906 (2012)

[42] A. Adriani, A. Bracco, D. Grassi, M.L. Moriconi, A. Mura, G. Orton, F. Altieri, A., Ingersoll, J. Geophys. Res.: Planets, 125, e2019JE006098 (2020) https://doi.org/10.1029/2019JE006098

[43] M.L. Moriconi, A. Migliorini, F. Altieri, A. Adriani, A. Mura, G. Orton, J.I. Lunine, D. Grassi et al., J. Geophys. Res.: Planets, 125, e2019JE006096 (2020) https://doi.org/10.1029/2019JE006096

[44] J. Harrington, R. G. French and K. Matcheva, ApJ., 716, $404(2010)$ 